\title{
ADAPAR-Studie: Ergebnisse zu Nahrungsmittelallergien nach fünf Jahren
}

\author{
Schlüsselwörter \\ Allergische Erkrankungen · Geburtskohorte . \\ Nahrungsmittelallergie · Toleranz
}

\begin{abstract}
Zusammenfassung
Hintergrund: Obwohl Nahrungsmittelallergien (NA) in vielen Fällen eine vorübergehende Erkrankung im Kindesalter sind, haben viele verschiedene Faktoren Einfluss darauf, wann bei wem eine NA auftritt. In der vorliegenden Studie analysierten wir die 5-JahresDaten von 33 Kindern, die im Rahmen der Geburtskohortenstudie ADAPAR (Adana Pediatric Allergy Research) im Süden der Türkei mit NA disgnostiziert wurden.
\end{abstract}

Methoden: 33 Säuglinge, bei denen bis zum Ende des ersten Lebensjahres im Rahmen der ADAPAR-Studie eine NA festgestellt wurde, wurden bis zum Alter von 5 Jahren alle 6 Monate untersucht. Die Untersuchungen umfassten jeweils eine klinische Beurteilung, eine Befragung, eine Blutprobennahme und einen Prick-Test.

Ergebnisse: Als auslösende Allergene wurden Kuhmilch ( $n=20)$, Eier $(n=17)$, Hühnerfleisch $(n=1)$ und Bananen $(n=1)$ identifiziert. Von den 17 Patienten mit Eiallergie entwickelten 14 Patienten vollständige Toleranz und ein Patient partielle Toleranz (d.h. Tole- ranz gegenüber gebackenen Nahrungsmitteln). Von den 20 Patienten mit Milchallergie trat bei 16 Patienten vollständige Toleranz ein und bei einem Patient partielle Toleranz. Das mittlere Alter bei Eintritt der Toleranz gegenüber Ei betrug 22,4 \pm 7,5 Monate, die Toleranz gegenüber Kuhmilch trat mit 20,9 \pm 1,1 Monaten ein. Der Patient mit Allergie gegen Hühnerfleisch und der Patient mit Allergie gegen Bananen entwickelten vollständige Toleranz. Auch andere allergische Erkrankungen wurden festgestellt: allergische Rhinitis bei 27,2\%, atopische Dermatitis bei 21,2\%, Asthma bei 9\%, Urtikaria bei 9\% und Arzneimittelallergien bei 9\%.

Schlussfolgerungen: Unsere Ergebnisse bestätigen frühe und hohe Toleranzraten noch vor Erreichung des Schulalters bei Kindern mit NA, die bereits im Säuglingsalter aufgetreten sind. Diese Ergebnisse helfen Kinderärzten dabei, Eltern von Säuglingen mit Kuhmilch- oder Hühnereiallergie kompetent zu beraten.

C 2016 S. Karger GmbH, Freiburg

\section{Originalartikel \\ The ADAPAR Birth Cohort Study: Food Allergy Results at Five Years and New Insights}

Dilek Doğruel Gülbin Bingöl Mustafa Yılmaz Derya Ufuk Altıntaş

Department of Pediatric Allergy and Immunology, Çukurova University Faculty of Medicine, Adana, Turkey 
Prof. Dr. Regina Fölster-Holst

rfoelsterholst@dermatology.uni-kiel.de

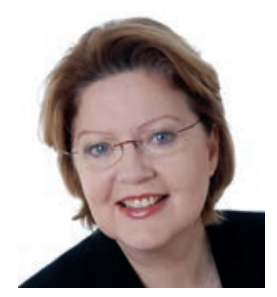

\section{Relevanz für den deutschen Praxis- und Klinikalltag}

Nahrungsmittel sind als Provokationsfaktoren der atopischen Dermatitis bekannt. In der infantilen Phase kommt den Grundnahrungsmitteln Milch und Hühnerei die größte Bedeutung zu. Während es sich hier in den meisten Fällen um transiente Allergien handelt, sind die pollenassoziierten Nahrungsmittelallergien (orales Allergiesyndrom bei Kern- und Steinobstallergie) eher durch Persistenz gekennzeichnet und weisen sich als Provokationsfaktor des atopischen Ekzems im späteren Kindes- und Erwachsenenalter aus. Ist die Nahrungsmittelallergie in der frühen Kindheit durch Provokationstestungen oder differenzierte anamnestische Angaben der Eltern in Verbindung mit Blut- und/oder Prick-Test-ergebnissen gesichert, sollten die betreffenden Nahrungsmittel zunächst gemieden werden. Das gilt nicht für die Primärprävention, bei der das Immunsystem des Darms mit den Nahrungsmitteln in Kontakt getreten sein sollte, damit sich eine Toleranz gegenüber den Allergenen entwickeln kann. Die Autoren des Artikels haben im Rahmen einer Geburtenkohorte am Ende des 1. Lebensjahres anhand von Provokationstestungen bei 33 von insgesamt 1377 Säuglingen eine Nahrungsmittelallergie diagnostiziert. Bei 28 von 33 war diese Allergie transienter Natur, wie bei den Follow-up-Untersuchungen festgestellt wurde. Für die Praxis hat das Konsequenzen: Erstens sollte den Eltern die bekannte hohe Abheilungsrate mitgeteilt werden. Zweitens sollten Follow-up-Untersuchungen mit Provokationstestungen erfolgen, die dann bei Toleranz die Karenz beenden können.

\section{Neue Erkenntnisse}

Die Autoren zeigen eine hohe Toleranzrate auf, die sie auf folgende Faktoren zurückführen: frühe Diagnosestellung der Nahrungsmittelallergie und somit Einleitung einer Karenz, verlängertes Stillen >6 Monate, exklusives Stillen für 4 Monate, Einführung der Beikost nach dem 4. Lebensmonat sowie Präund/oder Probiotika-Gaben.

\section{Kritik}

Die Gabe von Prä- und/oder Probiotika gehört (noch) nicht zu den Primär- und Sekundärpräventionen atopischer Erkrankun- gen. Die Datenlage hierzu ist immer noch kontrovers, so dass noch keine allgemeinen Empfehlungen abgegeben werden können.

Wie die Autoren selbst feststellen, ist bei einer Patientenzahl von 33 die Aussagekraft der Ergebnisse begrenzt. Zudem wurden Patienten, bei denen die Allergie bei der ersten Follow-up-Untersuchung bereits verschwunden war, nicht weiter verfolgt. Damit gehen Patienten, die im weiteren Verlauf gegebenenfalls erneut eine Nahrungsmitteallergie entwickeln, für die Auswertung verloren. Es fehlen detaillierte Ergebnisse zu Anamnese (Eigen- und Familienanamnese), klinischer Untersuchung, Laborparametern und auch zu klinischen Symptomen nach Provokationstestungen.

\section{Perspektiven für die Behandlungspraxis}

Die Untersuchung hat gute Ansätze, die in größeren prospektiven Untersuchungen verfolgt werden sollten. Dabei sollte besonders die folgende Frage beantwortet werden: Können Prä- und/oder Probiotika die Entwicklung atopischer Erkrankungen verhindern oder bei bereits manifesten Erkrankungen die Symptome lindern, wie es In-vitro-Studien erwarten lassen?

Durch Eingrenzung des «kritischen Zeitfensters» könnten für Interventionen Studien initiiert werden, die Nahrungsmittel gezielt zur Toleranzentwicklung des intestinalen Immunsystems einführen.

\section{Referenzen}

1 Lee SE, Kim H: Update on early nutrition and food allergy in children Yonsei Med J 2016;57:542-548.

2 Jennings S, Prescott SL: Early dietary exposures and feeding practices: role in pathogenesis and prevention of allergic disease? Postgrad Med 2010;86:94-99.

3 Longo G, Berti I, Burks AW, Krauss B, Barbi E: IgE-mediated food allergy in children. Lancet 2013;382:1656-1664.

4 Fölster-Holst R: Probiotics in the treatment and prevention of atopic dermatitis. Ann Nutr Metab 2010;57(suppl 1):16-19.

Kontaktadresse: Prof. Dr. Regina Fölster-Holst, Klinik für Dermatologie, Venerologie und Allergologie, Universitätsklinikum Schleswig-Holstein, Campus Kiel, Arnold-Heller-Straße 3, Haus 19, 24105 Kiel, Deutschland 


\section{UK \\ C UNIVERSITÄTSKLINIKUM \\ Schleswig-Holstein}

für Kinderdermatologie (European Society for Pediatric

Dermatology, ESPD).

Die Kieler Dermatologin Professorin Dr. Regina Fölster-Holst, Klinik für Dermatologie, Venerologie und Allergologie, Medizinische Fakultät der Christian-Albrechts-Universität zu Kiel (CAU) und Universitätsklinikum Schleswig-Holstein (UKSH) wurde bei der 13. Jahrestagung der ESPD für die nächsten zwei Jahre ins Amt gewählt.

«Die Interaktion von Wissenschaft und Praxis ist besonders wichtig, um den hautkranken Kindern eine optimale Therapie zukommen zu lassen», sagt Fölster-Holst. Ihre wissenschaftlichen Schwerpunkte sind die Kinderdermatologie, Neurodermitis und Allergien sowie Parasitosen. Das Hauptaugenmerk dabei ist die Neurodermitis im Kindesalter, bei der sie unterschiedliche klinische Erscheinungsformen hinsichtlich immunologischer und genetischer Faktoren sowie der Hautbarriere untersucht. Sie erhofft sich dadurch eine gezieltere und optimale Behandlung.

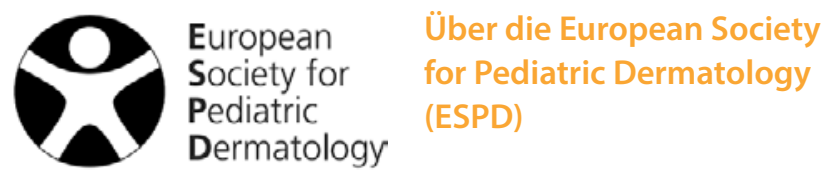

Die ESPD ist eine unabhängige wissenschaftliche Gesellschaft, die sich an Fachleute aus den Bereichen Dermatologie und Pädiatrie richtet. Ziel der ESPD ist es, die klinische Versorgung im Fachbereich der Kinderdermatologie zu verbessern und interdisziplinäre Forschungsprojekte sowie die Ausbildung zu fördern. 